\title{
EIGENVALORES DE UNA MATRIZ LAPLACIANA
}

\section{EIGENVALUES OF A LAPLACIAN MATRIX}

\author{
RUBÉN A. MARTÍNEZ-AVENDAÑO
}

\begin{abstract}
RESUMEN. En esta nota se presenta un método para calcular los eigenvalores de una matriz laplaciana. Este cálculo se lleva a cabo primero obteniendo una expresión conveniente para el polinomio característico utilizando funciones generadoras. Posteriormente, utilizando esta expresión, se obtienen los eigenvalores.
\end{abstract}

ABSTRACT. In this note, we show how to calculate eigenvalues of a laplacian matriz. This computation is done by first obtaining a useful expression for the characteristic polynomial using generating functions. With this expression, we obtain the eigenvalues.

Palabras clave: Laplaciano discreto, eigenvalores, solución numérica de ecuaciones diferenciales, función generadora.

Keywords: Discrete Laplacian, eigenvalues, numerical solution of differential equations, generating function.

\section{INTRODUCCIÓN}

En diversas áreas de las matemáticas aplicadas, uno se enfrenta con el problema de resolver la llamada ecuación de Poisson. Por ejemplo, en física se utiliza esta ecuación para modelar fenómenos de difusión, electroestática, campos gravitacionales, etc. La ecuación de Poisson es usada en otras muchas áreas, como en procesamiento y compresión de imágenes, en ingeniería mecánica, etc. Además, el problema de Poisson es un ejemplo básico muy estudiado en ecuaciones diferenciales y que sirve de trampolín para estudiar problemas de ecuaciones diferenciales elipticas.

El problema de Poisson (con condiciones de Dirichlet) consiste en lo siguiente. Dado un conjunto $\Omega \in \mathbb{R}^{n}$ (usualmente abierto, conexo y acotado) y dada una función $f: \Omega \rightarrow \mathbb{R}$, queremos encontrar una función $u$, con dominio la cerradura de $\Omega$ tal que

$$
-\Delta u=f \quad \text { y } \quad u(x)=0 \quad \text { para } x \text { en la frontera de } \Omega .
$$

Aquí $\Delta$ es el operador definido como

$$
\Delta u:=\frac{\partial^{2} u}{\partial x_{1}^{2}}+\frac{\partial^{2} u}{\partial x_{2}^{2}}+\cdots+\frac{\partial^{2} u}{\partial x_{n}^{2}} .
$$


Al operador $-\Delta$ se la conoce como el operador laplaciano. En esta nota nos olvidaremos del signo menos, pues no tiene relevancia para lo que sigue y este se puede pasar al otro lado de la ecuación.

Consideremos la versión del problema en una dimensión. Dada $f:(0,1) \rightarrow \mathbb{R}$ hay que encontrar una función $u:[0,1] \rightarrow \mathbb{R}$ tal que

$$
u^{\prime \prime}(x)=f(x) \quad \text { para todo } x \in(0,1)
$$

y $u(0)=0=u(1)$.

Por supuesto, la manera «fácil» de resolver este problema es «integrar dos veces»y utilizar las condiciones de frontera para encontrar las constantes de integración adecuadas. Desafortunadamente, muchas veces puede ser muy difícil (e incluso imposible) integrar explícitamente. Es por esto que queremos resolver el problema numéricamente.

En esta nota, explicaremos brevemente cómo se puede hacer este proceso de discretización, del cual surge una matriz que llamamos «matriz laplaciana». Sin embargo, nuestro objetivo principal es mostrar un método conocido, pero pocas veces mostrado a alumnos de licenciatura, acerca de cómo encontrar los eigenvalores de esta matriz laplaciana, los cuales son útiles en diversas aplicaciones. Trataremos de mostrar estos métodos de la manera más «elemental» posible (por «elemental» no queremos decir «fácil», sino que no utilice resultados avanzados).

Vale la pena aclarar que el concepto de matriz laplaciana que mostramos aquí no es el mismo (pero sí está relacionado) con la matriz laplaciana de una gráfica. Para más información sobre estos interesantes temas recomendamos al lector consulte Cvetković et al (2010).

\section{UNA MATRIZ LAPLACIANA}

Una manera de resolver el problema de Poisson es discretizando. Sea $n \in \mathbb{N}$ y dividamos el intervalo [0,1] en $n+1$ subintervalos iguales. Es decir, consideramos la partición del intervalo $[0,1]$ dada por

$$
\left\{0, \frac{1}{n+1}, \frac{2}{n+1}, \frac{3}{n+1}, \ldots, \frac{n}{n+1}, 1\right\} .
$$

En concreto, sea

$$
x_{k}:=\frac{k}{n+1}
$$

cada uno de los puntos de la partición y definamos $u_{k}:=u\left(x_{k}\right)$ y $f_{k}:=f\left(x_{k}\right)$ para $k=0,1,2, \ldots, n+1$.

Una manera de aproximar la segunda derivada, usando diferencias finitas, es por medio de la ecuación

$$
u^{\prime \prime}(a) \simeq \frac{u(a+h)-2 u(a)+u(a-h)}{h^{2}},
$$

donde $h$ se asume pequeño (véase, por ejemplo, Burden et al (2001), pp. 660-662 o Kincaid et al (2002), pp. 465-469). En nuestro caso $h=1 /(n+1)$. 
Aplicando la fórmula anterior a la ecuacion (1) en cada uno de los puntos $\left\{x_{1}, x_{2}, \ldots, x_{n}\right\}$ se obtiene el siguiente sistema de $n$ ecuaciones:

$$
\begin{aligned}
\frac{u_{0}-2 u_{1}+u_{2}}{h^{2}} & =f_{1} \\
\frac{u_{1}-2 u_{2}+u_{3}}{h^{2}} & =f_{2} \\
\frac{u_{2}-2 u_{3}+u_{4}}{h^{2}} & =f_{3} \\
& \vdots \\
\frac{u_{n-1}-2 u_{n}+u_{n+1}}{h^{2}} & =f_{n} .
\end{aligned}
$$

Recordamos ahora las condiciones de Dirichlet $u(0)=1$ y $u(1)=0$, es decir, $u_{0}=0$ y $u_{n+1}=0$. Usando esto, el sistema anterior se escribe en forma matricial como

$$
\left(\begin{array}{cccccccc}
-2 & 1 & 0 & 0 & 0 & \ldots & 0 & 0 \\
1 & -2 & 1 & 0 & 0 & \ldots & 0 & 0 \\
0 & 1 & -2 & 1 & 0 & \ldots & 0 & 0 \\
0 & 0 & 1 & -2 & 1 & \ldots & 0 & 0 \\
0 & 0 & 0 & 1 & -2 & \ldots & 0 & 0 \\
\vdots & \vdots & & & & & \vdots & \vdots \\
0 & 0 & 0 & 0 & 0 & \ldots & -2 & 1 \\
0 & 0 & 0 & 0 & 0 & \ldots & 1 & -2
\end{array}\right)\left(\begin{array}{c}
u_{1} \\
u_{2} \\
u_{3} \\
u_{4} \\
u_{5} \\
\vdots \\
u_{n-1} \\
u_{n}
\end{array}\right)=h^{2}\left(\begin{array}{c}
f_{1} \\
f_{2} \\
f_{3} \\
f_{4} \\
f_{5} \\
\vdots \\
f_{n-1} \\
f_{n}
\end{array}\right) .
$$

El problema de Poisson discreto, con condiciones de Dirichlet, consiste en resolver el sistema anterior. Es decir, si dada $f:(0,1) \rightarrow \mathbb{R}$, podemos resolver el sistema anterior, entonces los puntos $\left\{u_{1}, u_{n}, \ldots, u_{n}\right\}$ aproximan al valor de la solución $u$ de la ecuación (1) en los puntos $x_{1}, x_{2}, \ldots, x_{n}$. Una vez hecho esto, se pueden usar splines cúbicos (véase, por ejemplo, Burden et al (2001)) para aproximar el valor de $u$ en otros puntos.

Llamaremos a la matriz

$$
L_{n}:=\left(\begin{array}{cccccccc}
-2 & 1 & 0 & 0 & 0 & \ldots & 0 & 0 \\
1 & -2 & 1 & 0 & 0 & \ldots & 0 & 0 \\
0 & 1 & -2 & 1 & 0 & \ldots & 0 & 0 \\
0 & 0 & 1 & -2 & 1 & \ldots & 0 & 0 \\
0 & 0 & 0 & 1 & -2 & \ldots & 0 & 0 \\
\vdots & \vdots & & & & & \vdots & \vdots \\
0 & 0 & 0 & 0 & 0 & \ldots & -2 & 1 \\
0 & 0 & 0 & 0 & 0 & \ldots & 1 & -2
\end{array}\right)
$$

la matriz laplaciana discreta.

Por supuesto, uno está interesado en las propiedades de $L_{n}$. Una de las preguntas más naturales es: ¿cuáles son sus eigenvalores? En el resto de esta nota, describiremos un método para encontrarlos. 


\section{Polinomio característico}

Para encontrar los eigenvalores de $L_{n}$ existen varios métodos. Uno de los más populares y que se encuentra en varios libros de texto (e.g. Strang, 2007) es «adivinar» los eigenvectores, evaluar $L_{n}$ en ellos y de ahí obtener los eigenvalores. ${ }^{\ddagger}$

Nos parece que este método, aunque fácil, es engañoso. ¿Cómo se encontraron estos eigenvectores? Usualmente estos se «adivinan» por consideraciones físicas o de otra índole. Es por esto que nuestro objetivo en esta nota es mostrar un método que encuentra los eigenvalores de $L_{n}$ sin necesidad de «adivinarlos». Por supuesto, se requiere trabajo adicional.

Sea $A_{n}$ la matriz $n \times n$ dada por

$$
A_{n}:=\left(\begin{array}{cccccccc}
0 & 1 & 0 & 0 & 0 & \ldots & 0 & 0 \\
1 & 0 & 1 & 0 & 0 & \ldots & 0 & 0 \\
0 & 1 & 0 & 1 & 0 & \ldots & 0 & 0 \\
0 & 0 & 1 & 0 & 1 & \ldots & 0 & 0 \\
0 & 0 & 0 & 1 & 0 & \ldots & 0 & 0 \\
\vdots & \vdots & & & & & \vdots & \vdots \\
0 & 0 & 0 & 0 & 0 & \ldots & 0 & 1 \\
0 & 0 & 0 & 0 & 0 & \ldots & 1 & 0
\end{array}\right) .
$$

Si $I_{n}$ denota a la matriz identidad $n \times n$, notemos que $L_{n}=A_{n}-2 I_{n}$. De aquí se sigue que $\lambda$ es un eigenvalor de $L_{n}$ si y solo si $\lambda+2$ es un eigenvalor de $A_{n}$. Es por esto que nos concentraremos primero en encontrar los eigenvalores de $A_{n}$ (lo cual es más fácil, pues $A_{n}$ es una matriz más «sencilla» que $L_{n}$ ). Para esto, trataremos de encontrar una expresión explícita para el polinomio característico de $A_{n}$.

Definamos $p_{n}(x):=\operatorname{det}\left(x I_{n}-A_{n}\right)$. Claramente,

$$
p_{1}(x)=x \quad \text { y } \quad p_{2}(x)=x^{2}-1 .
$$

Obsérvese que para $n \geq 3$ se tiene

$$
p_{n}(x)=\operatorname{det}\left(x I_{n}-A_{n}\right)=\left(\begin{array}{c|c|cccccc}
x & -1 & 0 & 0 & 0 & \ldots & 0 & 0 \\
\hline-1 & x & -1 & 0 & 0 & \ldots & 0 & 0 \\
\hline 0 & -1 & x & -1 & 0 & \ldots & 0 & 0 \\
0 & 0 & -1 & x & -1 & \ldots & 0 & 0 \\
0 & 0 & 0 & -1 & x & \ldots & 0 & 0 \\
\vdots & \vdots & & & & & \vdots & \vdots \\
0 & 0 & 0 & 0 & 0 & \ldots & x & -1 \\
0 & 0 & 0 & 0 & 0 & \ldots & -1 & x
\end{array}\right)
$$

${ }^{\ddagger}$ Otro método consiste en desarrollar teoremas sobre eigenvalores de matrices de adyacencia de gráficas y aplicarlos a una gráfica partícular, como se hace en Cvetković et al, 2010, p. 47. 
y expandiendo por menores a lo largo del primer renglón, obtenemos

$$
p_{n}(x)=x \operatorname{det}\left(\begin{array}{ccccccc}
x & -1 & 0 & 0 & \ldots & 0 & 0 \\
-1 & x & -1 & 0 & \ldots & 0 & 0 \\
0 & -1 & x & -1 & \ldots & 0 & 0 \\
0 & 0 & -1 & x & \ldots & 0 & 0 \\
\vdots & & & & & \vdots & \vdots \\
0 & 0 & 0 & 0 & \ldots & x & -1 \\
0 & 0 & 0 & 0 & \ldots & -1 & x
\end{array}\right)-(-1) \operatorname{det}\left(\begin{array}{cccccccc}
-1 & -1 & 0 & 0 & \ldots & 0 & 0 \\
\hline 0 & x & -1 & 0 & \ldots & 0 & 0 \\
0 & -1 & x & -1 & \ldots & 0 & 0 \\
0 & 0 & -1 & x & \ldots & 0 & 0 \\
\vdots & & & & & \vdots & \vdots \\
0 & 0 & 0 & 0 & \ldots & x & -1 \\
0 & 0 & 0 & 0 & \ldots & -1 & x
\end{array}\right) .
$$

Pero, expandiendo el segundo determinante del lado derecho a lo largo de la primera columna obtenemos

$$
p_{n}(x)=x \operatorname{det}\left(x I_{n-1}-A_{n-1}\right)-\operatorname{det}\left(x I_{n-2}-A_{n-2}\right) ;
$$

es decir, para $n \geq 3$, obtenemos la relación

$$
p_{n}(x)=x p_{n-1}(x)-p_{n-2}(x) .
$$

Utilizando la relación de arriba, y el hecho de que $p_{1}(x)=x$ y $p_{2}(x)=x^{2}-1$ obtenemos la siguiente lista de polinomios característicos

$$
\begin{aligned}
p_{1}(x) & =x \\
p_{2}(x) & =x^{2}-1 \\
p_{3}(x) & =x^{3}-2 x \\
p_{4}(x) & =x^{4}-3 x^{2}+1 \\
p_{5}(x) & =x^{5}-4 x^{3}+3 x \\
p_{6}(x) & =x^{6}-5 x^{4}+6 x^{2}-1 \\
p_{7}(x) & =x^{7}-6 x^{5}+10 x^{3}-4 x \\
p_{8}(x) & =x^{8}-7 x^{6}+15 x^{4}-10 x^{2}+1 \\
p_{9}(x) & =x^{9}-8 x^{7}+21 x^{5}-20 x^{3}+5 x \\
p_{10}(x) & =x^{10}-9 x^{8}+28 x^{6}-35 x^{4}+15 x^{2}-1 .
\end{aligned}
$$

No es tan complicado encontrar un patrón para la forma general de estos polinomios. De hecho, como veremos en el apéndice, estos polinomios se obtienen haciendo un cambio de variables de una familia conocida y bien estudiada de polinomios de los cuales se conocen sus raíces.

Nuestro proposito aquí, sin embargo, es obtener una expresión de estos polinomios que no utilice resultados avanzados y utilizarla para encontrar las raíces de cada polinomio $p_{n}$.

\section{FunCión GENERAdora}

Un método que se utiliza para encontrar formas cerradas de funciones definidas recursivamente es el de las funciones generadoras. Considérese la expresión

$$
p_{n}(x)=x p_{n-1}(x)-p_{n-2}(x),
$$


la cual es válida para todo $n \geq 3$ y donde pensaremos que $x$ es ahora un parámetro real. Multiplicando por $t^{n}$ y haciendo la suma formal (es decir, olvidándonos de cualquier cuestión de convergencia) desde $n=3$ hasta $n=\infty$ se obtiene

$$
\sum_{n=3}^{\infty} p_{n}(x) t^{n}=x \sum_{n=3}^{\infty} p_{n-1}(x) t^{n}-\sum_{n=3}^{\infty} p_{n-2}(x) t^{n} .
$$

Si definimos la función

$$
g_{x}(t):=\sum_{n=1}^{\infty} p_{n}(x) t^{n}
$$

la expresión anterior se puede escribir como

$$
g_{x}(t)-p_{1}(x) t-p_{2}(x) t^{2}=x t\left(g_{x}(t)-p_{1}(x) t\right)-t^{2} g_{x}(t) .
$$

Agrupando $g_{x}$ se obtiene

$$
g_{x}(t)\left(1-x t+t^{2}\right)=p_{1}(x) t+p_{2}(x) t^{2}-x t\left(p_{1}(x) t\right),
$$

Utilizando el hecho que $p_{1}(x)=x$ y $p_{2}(x)=x^{2}-1$ esta expresión se simplifica a

$$
g_{x}(t)\left(1-x t+t^{2}\right)=x t+\left(x^{2}-1\right) t^{2}-x t x t,
$$

de donde se despeja $g_{x}$ para obtener

$$
g_{x}(t)=\frac{x t-t^{2}}{1-x t+t^{2}} .
$$

Escribamos esta expresión como

$$
g_{x}(t)=\frac{1}{1-x t+t^{2}}-1
$$

y encontremos su serie de potencias en $t$.

Sean $a_{+}(x)$ y $a_{-}(x)$ las dos raíces del polinomio $1-x t+t^{2}$. Estas raíces son reales y distintas si $|x|>2$, son reales e iguales si $|x|=2$ y son distintas, complejas y conjugadas si $|x|<2$. De cualquier manera se tiene que

$$
\left(t-a_{+}(x)\right)\left(t-a_{-}(x)\right)=t^{2}-x t+1 .
$$

Supongamos por un momento que las raíces son distintas: $a_{+}(x) \neq a_{-}(x)$ (esto es equivalente a $|x| \neq 2$ ). Definamos

$$
\gamma(x)=\frac{1}{a_{+}(x)-a_{-}(x)} .
$$

Separando usando sumas parciales, podemos escribir

$$
\frac{1}{t^{2}-x t+1}=\gamma(x)\left(\frac{1}{t-a_{+}(x)}-\frac{1}{t-a_{-}(x)}\right) .
$$

Utilizando la serie geométrica, obtenemos

$$
\frac{1}{t-a_{+}(x)}=-\sum_{n=0}^{\infty} \frac{t^{n}}{a_{+}(x)^{n+1}}
$$

$\mathrm{y}$

$$
\frac{1}{t-a_{-}(x)}=-\sum_{n=0}^{\infty} \frac{t^{n}}{a_{-}(x)^{n+1}}
$$


Por lo tanto,

$$
\frac{1}{t^{2}-x t+1}=\gamma(x)\left(-\sum_{n=0}^{\infty} \frac{t^{n}}{a_{+}(x)^{n+1}}+\sum_{n=0}^{\infty} \frac{t^{n}}{a_{-}(x)^{n+1}}\right)
$$

y de lo cual se sigue que

$$
\frac{1}{t^{2}-x t+1}=\gamma(x) \sum_{n=0}^{\infty}\left(\frac{1}{a_{-}(x)^{n+1}}-\frac{1}{a_{+}(x)^{n+1}}\right) t^{n}
$$

Como $a_{+}(x) a_{-}(x)=1$, esta expresión se simplifica a

$$
\frac{1}{t^{2}-x t+1}=\gamma(x) \sum_{n=0}^{\infty}\left(a_{+}(x)^{n+1}-a_{-}(x)^{n+1}\right) t^{n}
$$

y de aqui se sigue que

$$
g_{x}(t)=\sum_{n=1}^{\infty}\left(\frac{a_{+}(x)^{n+1}-a_{-}(x)^{n+1}}{a_{+}(x)-a_{-}(x)}\right) t^{n},
$$

Comparando las dos expresiones que tenemos para $g_{x}(t)$ se sigue que

$$
p_{n}(x)=\frac{a_{+}(x)^{n+1}-a_{-}(x)^{n+1}}{a_{+}(x)-a_{-}(x)} .
$$

Esta expresión es válida para $|x| \neq 2$.

Si el lector tiene dudas de la validez de esta última fórmula (y quizá debería, pues olvidarse de la convergencia no es tan sencillo), es fácil convencerse de su veracidad usando inducción. Hagámoslo. Sólo recuérdese que $a_{+}(x)+a_{-}(x)=x \mathrm{y}$ $a_{+}(x) a_{-}(x)=1$ para todo $x \in \mathbb{R}$.

Si $n=1$ se obtiene

$$
p_{1}(x)=\frac{a_{+}(x)^{2}-a_{-}(x)^{2}}{a_{+}(x)-a_{-}(x)}=a_{+}(x)+a_{-}(x)=x .
$$

Para $n=2$ se obtiene

$$
\begin{aligned}
p_{2}(x) & =\frac{a_{+}(x)^{3}-a_{-}(x)^{3}}{a_{+}(x)-a_{-}(x)} \\
& =a_{+}(x)^{2}+a_{+}(x) a_{-}(x)+a_{-}(x)^{2} \\
& =\left(a_{+}(x)+a_{-}(x)\right)^{2}-a_{+}(x) a_{-}(x) \\
& =x^{2}-1 .
\end{aligned}
$$


Inductivamente, para $n \geq 3$,

$$
\begin{aligned}
p_{n}(x) & =x p_{n-1}(x)-p_{n-2}(x) \\
& =x \frac{a_{+}(x)^{n}-a_{-}(x)^{n}}{a_{+}(x)-a_{-}(x)}-\frac{a_{+}(x)^{n-1}-a_{-}(x)^{n-1}}{a_{+}(x)-a_{-}(x)} \\
& =\frac{x a_{+}(x)^{n}-x a_{-}(x)^{n}-a_{+}(x)^{n-1}+a_{-}(x)^{n-1}}{a_{+}(x)-a_{-}(x)} \\
& =\frac{a_{+}(x)^{n-1}\left(x a_{+}(x)-1\right)-a_{-}(x)^{n-1}\left(x a_{-}(x)-1\right)}{a_{+}(x)-a_{-}(x)} \\
& =\frac{a_{+}(x)^{n-1}\left(a_{+}(x)^{2}\right)-a_{-}(x)^{n-1}\left(a_{-}(x)^{2}\right)}{a_{+}(x)-a_{-}(x)} \\
& =\frac{a_{+}(x)^{n+1}-a_{-}(x)^{n+1}}{a_{+}(x)-a_{-}(x)}
\end{aligned}
$$

ya que $a_{+}(x)$ y $a_{-}(x)$ son soluciones de la ecuación $x t-1=t^{2}$.

La expresión encontrada para $p_{n}$ no es válida para $|x|=2$. Sin embargo, se puede reescribir esta expresión como

$$
\frac{a_{+}(x)^{n+1}-a_{-}(x)^{n+1}}{a_{+}(x)-a_{-}(x)}=\sum_{k=0}^{n} a_{+}(x)^{n-k} a_{-}(x)^{k},
$$

la cual sí es válida para todo $x \in \mathbb{C}$. Por lo tanto, se tiene la siguiente expresión para el polinomio característico de $A_{n}$,

$$
p_{n}(x)=\sum_{k=0}^{n} a_{+}(x)^{n-k} a_{-}(x)^{k},
$$

la cual sí es válida para todo $x \in \mathbb{R}$.

\section{RAÍCES DEL POLINOMIO CARACTERÍSTICO}

Observémos que 2 y -2 no son raíces de $p_{n}$. De hecho, nótese primero que $a_{+}(2)=1=a_{-}(2)$. Por lo tanto

$$
p_{n}(2)=\sum_{k=0}^{n} a_{+}(2)^{n-k} a_{-}(2)^{k}=\sum_{k=0}^{n} 1^{n-k} 1^{k}=1^{n}(n+1) \neq 0 .
$$

Análogamente, usando el hecho que $a_{+}(-2)=-1=a_{-}(-2)$ se tiene que

$$
p_{n}(-2)=(-1)^{n}(n+1) \neq 0 .
$$

Por lo tanto, si queremos encontrar las raíces del polinomio $p_{n}(x)$ basta encontrar los ceros de la expresión

$$
\frac{a_{+}(x)^{n+1}-a_{-}(x)^{n+1}}{a_{+}(x)-a_{-}(x)} .
$$

Encontremos para que números $x \in \mathbb{C}$ se tiene

$$
a_{+}(x)^{n+1}-a_{-}(x)^{n+1}=0 .
$$

Claramente $a_{+}(x)^{n+1}=a_{-}(x)^{n+1}$ si y solo si $a_{+}(x)=\omega^{k} a_{-}(x)$, donde $\omega$ es la $(n+1)$-ésima raíz primitiva de la unidad dada por

$$
\omega=\exp (i 2 \pi /(n+1))
$$


y $k=0,1,2, \ldots, n$. Claramente podemos olvidarnos del caso $k=0$ pues entonces $a_{+}(x)=a_{-}(x)$, lo cual implicaría que $|x|=2$, lo cual ya hemos descartado.

Analicemos el caso $|x|<2$. En este caso, tenemos

$$
a_{+}(x)=\frac{x+i \sqrt{4-x^{2}}}{2}
$$

y

$$
a_{-}(x)=\frac{x-i \sqrt{4-x^{2}}}{2}
$$

Se sigue entonces que

$$
x+i \sqrt{4-x^{2}}=\omega^{k}\left(x-i \sqrt{4-x^{2}}\right)
$$

lo cual se simplifica a

$$
i \sqrt{4-x^{2}}\left(1+\omega^{k}\right)=x\left(\omega^{k}-1\right) .
$$

Elevando al cuadrado obtenemos

$$
\left(x^{2}-4\right)\left(1+\omega^{k}\right)^{2}=x^{2}\left(\omega^{k}-1\right)^{2}
$$

y despejando $x^{2}$ se tiene

$$
x^{2}=\frac{4\left(1+\omega^{k}\right)^{2}}{\left(1+\omega^{k}\right)^{2}-\left(1-\omega^{k}\right)^{2}},
$$

de donde

Simplificando se obtiene

$$
x^{2}=\frac{4\left(1+\omega^{k}\right)^{2}}{4 \omega^{k}} .
$$

$$
x^{2}=\frac{1+2 \omega^{k}+\omega^{2 k}}{\omega^{k}}=\overline{\omega^{k}}+2+\omega^{k}=2+2 \cos \left(\frac{2 \pi k}{n+1}\right) .
$$

Utilizando la identidad $\cos (2 \theta)=2 \cos ^{2}(\theta)-1$, la expresión anterior se puede escribir como

$$
x^{2}=2+2 \cos \left(\frac{2 \pi k}{n+1}\right)=2+2\left(2 \cos ^{2}\left(\frac{\pi k}{n+1}\right)-1\right)=4 \cos ^{2}\left(\frac{\pi k}{n+1}\right) .
$$

Por lo que

$$
x= \pm 2 \cos \left(\frac{\pi k}{n+1}\right)
$$

con $k=1,2,3, \ldots, n$.

Obsérvese que hay valores repetidos, ya que

$$
\cos \left(\frac{\pi k}{n+1}\right)=-\cos \left(\frac{\pi(n+1-k)}{n+1}\right)
$$

Por lo tanto, solamente hay $n$ valores diferentes correspondientes a

$$
x=2 \cos \left(\frac{\pi k}{n+1}\right) \text {, }
$$

para $k=1,2, \ldots, n$.

Como $p_{n}$ es un polinomio de grado $n$, estas son todas las raíces posibles y no hay raices con valor absoluto mayor que 2 .

Hemos demostrado el siguiente teorema. 
Teorema 1. Sea $A_{n}$ la matriz compleja de tamaño $n \times n$ dada por

$$
\left(\begin{array}{cccccccc}
0 & 1 & 0 & 0 & 0 & \ldots & 0 & 0 \\
1 & 0 & 1 & 0 & 0 & \ldots & 0 & 0 \\
0 & 1 & 0 & 1 & 0 & \ldots & 0 & 0 \\
0 & 0 & 1 & 0 & 1 & \ldots & 0 & 0 \\
0 & 0 & 0 & 1 & 0 & \ldots & 0 & 0 \\
\vdots & \vdots & & & & & \vdots & \vdots \\
0 & 0 & 0 & 0 & 0 & \ldots & 0 & 1 \\
0 & 0 & 0 & 0 & 0 & \ldots & 1 & 0
\end{array}\right) .
$$

Entonces, los eigenvalores de $A_{n}$ son los $n$ valores distintos dados por

$$
\left\{2 \cos \left(\frac{\pi k}{n+1}\right): k=1,2, \ldots, n\right\}
$$

Como $L_{n}=A_{n}-2 I_{n}$, obtenemos el siguiente teorema como corolario del anterior.

Teorema 2. Sea $L_{n}$ la matriz compleja de tamaño $n \times n$ dada por

$$
\left(\begin{array}{cccccccc}
-2 & 1 & 0 & 0 & 0 & \ldots & 0 & 0 \\
1 & -2 & 1 & 0 & 0 & \ldots & 0 & 0 \\
0 & 1 & -2 & 1 & 0 & \ldots & 0 & 0 \\
0 & 0 & 1 & -2 & 1 & \ldots & 0 & 0 \\
0 & 0 & 0 & 1 & -2 & \ldots & 0 & 0 \\
\vdots & \vdots & & & & & \vdots & \vdots \\
0 & 0 & 0 & 0 & 0 & \ldots & -2 & 1 \\
0 & 0 & 0 & 0 & 0 & \ldots & 1 & -2
\end{array}\right) .
$$

Entonces, los eigenvalores de $L_{n}$ son los $n$ valores distintos dados por

$$
\left\{-2+2 \cos \left(\frac{\pi k}{n+1}\right): k=1,2, \ldots, n\right\} .
$$

Nótese que, en particular, 0 nunca es un eigenvalor de $L_{n}$ y, por lo tanto, el problema de Poisson discreto con condiciones de Dirichlet tiene solución siempre.

\section{Apéndice: Polinomios de Chebyshev}

Como mencionamos anteriormente, los polinomios obtenidos en la sección 3 son una ligera modificacion de polinomios conocidos. Los polinomios de Chebyshev de segundo tipo (ver, por ejemplo, Burden et al, 2001 o Kincaid et al, 2002) se definen de manera recursiva por las relaciones $U_{0}(x):=1, U_{1}(x):=2 x$ y para $n \geq 2$ por

$$
U_{n}(x):=2 x U_{n-1}(x)-U_{n-2}(x) .
$$

Claramente $U_{2}(x):=4 x^{2}-1$. Nótese que $p_{1}(x)=U_{1}(x / 2)$, que $p_{2}(x)=U_{2}(x / 2)$ y que $p_{n}(x):=U_{n}(x / 2)$ satisface la relación

$$
p_{n}(x)=x p_{n-1}(x)-p_{n-2}(x) .
$$

Por lo tanto, los polinomios que encontramos en la sección 3 son exactamente

$$
p_{n}(x)=U_{n}(x / 2) .
$$

Una expresión alternativa para los polinomios de Chebyshev de segundo tipo es

$$
U_{n}(x)=\frac{\operatorname{sen}((n+1) \arccos (x))}{\operatorname{sen}(\arccos (x))} .
$$


para $x \in[-1,1]$. (Véase, por ejemplo, Kincaid et al (2002), p.48\%.

De aquí se pueden obtener fácilmente las raíces de la función $U_{n}(x / 2)$ resolviendo las ecuaciones

$$
(n+1) \operatorname{arc} \cos (x / 2)=\pi k,
$$

para $k \in \mathbb{Z}$. Se tiene entonces que $x=2 \cos \left(\frac{\pi k}{n+1}\right)$ para $k \in \mathbb{Z}$. Se obtienen $n+1$ valores diferentes correspondientes a $k=0,1,2, \ldots, n$. Sin embargo, nótese que el valor correspondiente a $k=0$ es $x=2$ y se tiene que

$$
p_{n}(2)=U_{n}(1)
$$

pero 1 no es raíz de $U_{n}$ : la culpa la tiene el denominador de $U_{n}$ el cual se anula en 1 pues

$$
\operatorname{sen}(\arccos (1))=\operatorname{sen}(0)=0 .
$$

\section{REFERENCIAS}

Richard L. Burden y J. Douglas Faires, Numerical Analysis, séptima edición, Brooks/Cole, 2001.

Dragoš Cvetković, Peter Rowlinson y Slobodan Simić, An Introduction to the Theory of Graph Spectra, Cambridge University Press, 2010.

David Kincaid y Ward Cheney, Numerical Analysis: Mathematics of Scientific Computing, tercera edición, Brooks/Cole, 2002.

Gilbert Strang, Computational Science and Engineering, Wellesley-Cambridge Press, 2007.

Centro de Investigación en Matemáticas, Universidad Autónoma del Estado de Hidalgo, Ciudad del Conocimiento, Carr. Pachuca-Tulancingo, km 4.5, Pachuca, Hidalgo, 42184, Mexico

E-mail address: rubeno71@gmail.com 